\title{
Ingesta de productos naturales o macrobióticos: una llamada de atención a la salud pública ${ }^{1}$
}

\author{
Patricia Hernández-Rivera ${ }^{2}$
}

INSTITUCIÓN: Universidad de Costa Rica

\section{ENSAYO}

Este trabajo aborda la importancia de prevenir el uso indiscriminado de productos naturales o macrobióticos, sus posibles efectos perjudiciales en la salud, así concienciar a profesionales en el área de la salud sobre la importancia de incluir este tema durante la historia médica. Se analiza el incremento en el uso de productos naturales o macrobióticos, las ventajas de la etnomedicina como un resurgimiento de la industria farmacéutica y las desventajas que acarrea este tipo de productos. Las hierbas ofrecen una alternativa terapéutica como la creación de nuevos fármacos, aunque poseen efectos secundarios que podrían arriesgar la salud de los pacientes.

Palabras clave: ajo, ginkgo-biloba, hierba-de-San-Juan, medicina-tradicional, plantas-medicinales.

\footnotetext{
${ }^{1}$ Fecha de recibido: 24 de marzo del 2017

Fecha de aceptación: 25 de mayo del 2017

${ }^{2}$ Odontóloga General. Máster en Administración de Instituciones Educativas. Facultad de Odontología, Universidad de Costa Rica.

Costa Rica. Correo electrónico: patricia.hernandezrivera@ucr.ac.cr
} 


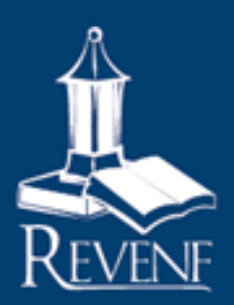

\title{
Revista Electrónica Enfermeria Actual en costa Rica
}

\section{Intake of natural or macrobiotic products: A wakeup call to public health ${ }^{1}$}

\author{
Patricia Hernández-Rivera ${ }^{2}$
}

INSTITUTION: University of Costa Rica

\section{ESSAY}

Prevention of the overuse of natural and macrobiotic products and possible adverse side effects as well as raising awareness among health professionals on the importance of including this subject while taking a medical history. An analysis about the increased usage of natural or macrobiotic products, the advantages of ethno-medicine as a resurgence of the pharmaceutical industry and the disadvantages of these types of products. Herbal medicine offers a therapeutic alternative such as the development of new drugs, although they may have side effects that could put at risk the health of patients.

Keywords: garlic, ginkgo-biloba, medicinal-plants, Saint-John-wort, traditional-medicine

\footnotetext{
${ }^{1}$ Date of receipt: March 24, 2017

Date of acceptance: May 25, 2017

${ }^{2}$ General Dentistry. Master in Administration of Educational Institutions. Faculty of Dentistry, University of Costa Rica. Costa Rica. E-mail: patricia.hernandezrivera@ucr.ac.cr
} 


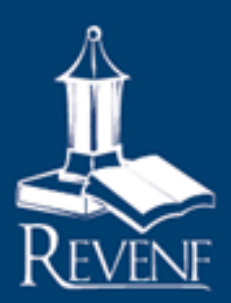

\title{
Revista Electrónica Enfermeria Actual en costa Rica
}

\section{Ingestão de produtos naturais ou macrobióticos: uma chamada de atenção à saúde pública ${ }^{1}$}

Instituição: Universidade de Costa Rica

\author{
Patricia Hernández-Rivera ${ }^{2}$
}

\begin{abstract}
ENSAIO
Este trabalho aborda a importância de prevenir o uso indiscriminado de produtos naturais ou macrobióticos, seus possíveis efeitos prejudiciais na saúde, e assim conscientizar aos profissionais na área da saúde sobre a importância de incluir este tema durante o historial médico. Analisa-se o incremento no uso de produtos naturais ou macrobióticos, as vantagens da etnomedicina como un ressurgimento da indústria farmacêutica e as desvantagens que acarreta este tipo de produtos. As plantas medicinais oferecem uma alternativa terapêutica como a criação de novos medicamentos, mesmo que possuam efeitos secundários que poderiam arriscar a saúde dos pacientes.
\end{abstract}

Palavras-chave: alho, ginkgo-biloba, erva-de-São João, medicina-tradicional, plantas-medicinais.

\footnotetext{
${ }^{1}$ Data de recebido: 24 de março de 2017

Data de aceitação: 25 de maio de 2017

${ }^{2}$ Odontóloga Geral. General. Mestre em Administração de Instituições Educacionais. Faculdade de Odontologia da Universidade de Costa Rica. Costa Rica. Correio electrónico: patricia.hernandezrivera@ucr.ac.cr
} 


\section{Revista Electrónica Enfermeria Actual en costa Rica}

\section{www.revenf.ucr.ac.cr}

\section{INTRODUCCIÓN}

En la actualidad tanto la población general como algunos autores han alabado las bondades de la medicina tradicional, de hondas raíces precolombinas, sobreviviente de la conquista, el colonialismo y el mestizaje, de la cual hay un acervo importante de conocimientos, que usualmente se transmiten de una generación a otra ${ }^{1}$. A lo anterior se suma que algunas empresas farmacológicas dedican importantes insumos a la investigación de los principios activos de la medicina natural, volcándose hacia una especie de reingeniería inversa ${ }^{2}$, hasta comprobar la importancia de esta.

Acorde con este pensamiento, un sector importante de la población, busca estilos de vida más sanos y eligen alimentos naturales, libres de químicos agregados y prefieren los productos macrobióticos a los alimentos procesados industrialmente: ese mismo público también se inclina por la medicina natural, la cual incluye productos herbarios frescos o secos e, incluso, parcialmente procesados, ingeridos como infusiones vegetales, cápsulas o en soluciones para uso oral. Este tipo de medicamento es culturalmente más aceptado, menos costoso y más accesible por no requerir de una receta médica, como ocurre con los medicamentos alopáticos ${ }^{1,3,4}$. Sin embargo, en un sector importante de la población, tal pensamiento se acompaña de la falsa premisa de que todo lo natural, incluyendo medicamentos, es inocuo, por lo tanto, puede tomarse sin ningún tipo de restricción ni dosificación, sin olvidar que la falta de un enfoque científico, termina atribuyéndoles más beneficios de los que realmente tienen. Lejos de esta creencia popular, este tipo de productos podrían causar efectos adversos e, inclusive, ocasionar interacciones farmacológicas que ponen en riesgo la salud del paciente ${ }^{5}$. Por esta razón, el objetivo de este ensayo es prevenir sobre el uso indiscriminado de productos naturales o macrobióticos, sus posibles efectos perjudiciales en la salud y concienciar a profesionales en el área de la salud sobre la importancia de incluir este tema durante la historia médica.

\section{DESARROLLO}

\section{Bondad y cuidados con la etnomedicina}

En torno al tema es importante acotar que gran parte de los medicamentos existentes tienen una base botánica, muchas veces extraída de la medicina popular. Aunque la lista es extensa, algunos ejemplos relevantes lo constituyen la quinina, extraída de la corteza del árbol de Cinchona sp posteriormente sintetizada en el laboratorio; también, así como el sauce blanco (Salix alba) del que se extrajo la aspirina y de la "dormilona" (Papaver somniferum) la morfina ${ }^{6}$.

Además, ha surgido el interés, y hasta cierto punto la necesidad, de encontrar nuevos principios farmacológicos para combatir infecciones resistentes a los antimicrobianos, acompañados de la esperanza de hallar tratamientos más efectivos y menos invasivos contra enfermedades actuales, motivo por el que la "medicina tradicional" se ha convertido en el foco de atención de grandes empresas farmacológicas y, con ello, han surgido distintos términos, como etnomedicina, etnobotánica, etnofarmacología, como fundamento de diversas investigaciones y publicaciones en este campo ${ }^{7}$. En esta misma línea de pensamiento nace la preocupación por la 


\section{Revista Electrónica Enfermeria Actual en costa Rica}

conservación de la flora, pues existe el riesgo de que especies en vías de extinción con potencial farmacológico no sean investigadas a tiempo.

Los fármacos extraídos de plantas se purifican y se someten a estudios exhaustivos y a ensayos clínicos para evaluar la efectividad y seguridad, antes de su comercialización. Esos estudios hacen la gran diferencia con los productos herbarios de la medicina popular, pues en esta se emplea extractos crudos, por lo tanto, pueden estar contaminados con otras sustancias químicas, algunas bioactivas, que causarían efectos perjudiciales o antagonizarían medicamentos, lo cual se desconoce ante la falta de análisis científicos y de certificados de calidad 6,8

Por otro lado, es engorroso estandarizar el contenido de los productos naturales, puesto que hay una alta variabilidad entre productores e, inclusive, diferencias importantes de un mismo producto de un lote a otro ${ }^{6}$. Asimismo, la cantidad de sustancias activas contenida en una planta depende de múltiples variables como el tiempo de cosecha, las condiciones climáticas, el tipo de suelo y la parte de la planta utilizada para la fabricación del producto ${ }^{8,9}$. Cuando este tipo de productos es parcialmente procesado y comercializado como "suplementos para la salud", pueden contener otros ingredientes que no son declarados en las etiquetas, además, del riesgo de contaminación por químicos como pesticidas, fertilizantes, toxinas y metales pesados, entre otras sustancias que podrían poner en riesgo la salud ${ }^{6,9}$.

Respecto de lo mencionado, es importante resaltar que tanto en la cultura oriental, como en la aborigen latinoamericana, se ha empleado la medicina herbaria por muchísimos años, aprovechando las propiedades benéficas de las plantas, inclusive se ha comprobado científicamente el efecto terapéutico y las propiedades antimicrobianas de algunas ${ }^{1}$ :un ejemplo es "la sangre de dragón", una secreción resinosa, de coloración rojiza, proveniente de varias especies de plantas, que en la medicina china -y también en la griega y romana- se utiliza para el tratamiento de múltiples dolencias; sin embargo, una serie de estudios, resumidos en la revisión de Gupta et al (2008), muestra la amplia gama de principios activos aislados de estas plantas, con lo cual la farmacología respalda la medicina tradicional, o viceversa ${ }^{10}$.

\section{El lado oscuro de la etnomedicina}

A parte de las bondades de las plantas medicinales, su empleo debe abordarse con cautela, debido a que la selección errónea de la hierba, la sobredosificación y la interacción con otros fármacos, puede generar complicaciones importantes ${ }^{6}$. Además, la falta de controles de calidad en su producción, cosecha y procesamiento, puede implicar variaciones importantes en la concentración de los principios activos de una determinada planta ${ }^{6}$. Asimismo, muchos de los beneficios por las que son recetadas, se basan en comentarios subjetivos, ausentes de criterios científicos de evaluación, asociados más por casualidad que por causalidad ${ }^{11}$, a lo que se agrega la existencia de plantas y hongos con toxinas, que fácilmente podrían envenenar o causar la muerte 11 .

Para ilustrar ese lado oscuro de algunas de las plantas medicinales de uso común en la cultura popular, a continuación, se señalan algunos ejemplos que muestran esos efectos negativos. 


\section{Revista Electrónica Enfermeria Actual en costa Rica}

\section{Ajo (Allium sativum)}

El ajo es una planta perenne bulbosa, comúnmente utilizada en la gastronomía. Aparte de la cocina, es importante en la medicina tradicional y entre sus propiedades médicas sobresale su acción antimicrobiana y su alto contenido en antioxidantes, que contribuyen con la reducción del estrés, del envejecimiento, prevención de enfermedades degenerativas, demencia, Alzheimer, disminución del colesterol, triglicéridos y la presión sanguínea, entre otros beneficios que engrosan la lista ${ }^{12}$. No obstante, es necesario reseñar sus propiedades científicamente probadas: posee un alto contenido de sustancias organosulfuradas: una de los principales es la aliína, la cual se convierte en alicina por acción de la alinasa, liberada cuando el ajo es triturado o cortado ${ }^{12,13,14}$; tal componente es autorreactivo y muy inestable, por lo que se convierte en otras sustancias con propiedades farmacológicas, como el ajoene, entre otros compuestos sulforados ${ }^{15,16,17}$. A continuación, se analiza algunos estudios, publicados en revistas científicas, que confirman muchos de los efectos beneficiosos que tradicionalmente se asocian con el ajo: la información al respecto es muy variada y extensa, pero aquí solo se referirá a algunos estudios, pues el interés está más enfocado en llamar la atención sobre el riesgo que acompaña su uso indiscriminado.

Las investigaciones más prometedoras son las referentes a los efectos antimicrobianos; especialmente cuando se enfocan en las cepas bacterianas resistentes a los antibióticos de uso regular, incluyendo aquellos de última generación en los cuales se deposita la esperanza para combatir las infecciones nosocomiales, tal es el caso de Staphylococcus aureus resistente a meticilina. En ese sentido, en estudios in vitro y en animales se ha documentado el efecto inhibitorio del extracto de alicina y el disulfuro de dialilo, producto de la reacción de la alicina, contra estas cepas ${ }^{18,17,20}$ y contra otras bacterias resistentes a antibióticos como Pseudomonas aeruginosa, Escherichia coli y Bacillus sp. ${ }^{21}$, también se ha demostrado su efecto contra Candida albicans ${ }^{17}$.

A pesar de lo anterior, no siempre los resultados obtenidos in vitro, se reflejan in vivo; un ejemplo fue la demostración de que los extractos de ajo inhiben el crecimiento de Campylobacter, pero al incorporar esos compuestos a la dieta de pollos no se registró una reducción en su colonización intestinal por esta bacteria ${ }^{22}$. No obstante, toda estudio sobre efectos antimicrobianos resulta prometedora en la era de la resistencia debido al uso indiscriminado de antibióticos.

Pero los efectos beneficiosos del ajo van más allá de sus propiedades antimicrobianas, como demuestra un estudio doble ciego, en el que los sujetos de prueba consumieron dos tabletas diarias de ajo, con $1.5 \mathrm{mg}$ de alicina y luego de 15 días habían mostrado una reducción significativa en la grasa corporal, sin embargo, después de ese periodo no mejoró el efecto ${ }^{23}$. Asimismo, el ajo tiene propiedades que evitan las enfermedades cardiovasculares por medio de relajación del músculo liso endotelial, provocando la expansión de los vasos y con ello, la disminución en la presión arterial, esto se cree que ocurre por dos mecanismos. en el primero, la alicina al ser una molécula que se descompone rápidamente por oxidación, libera sulfuro de hidrógeno, el cual es un potente vasodilatador y el otro, es que las moléculas de polisulfuros mejoran el balance óxido-reducción, en la regulación del óxido nítrico, el cual también es un vasodilatador ${ }^{12,16,17}$. Además, algunos estudios han demostrado que disminuye los triglicéridos y el conteo de colesterol de lipoproteínas de baja densidad sin alterar las lipoproteínas de alta densidad ${ }^{14,16,17}$.

Sin embargo, el ajo también tiene su efecto negativo: es un potente antiplaquetario, pues inhibe la agregación plaquetaria por medio de la inhibición del tromboxano A2, de la actividad de la fosfolipasa, la 


\section{Revista Electrónica Enfermeria Actual en costa Rica}

agregación inducida por epinefrina y la agregación inducida por calcio ionóforo, el cual moviliza el calcio intraplaquetario; además, incrementa la fibrinólisis ${ }^{24}$. Estas propiedades antiplaquetarias han ocasionado sangrados posoperatorios importantes, tal como ha sido documentado por Erian y McLaren (2013), quienes divulgaron el caso de una paciente de 37 años, sin antecedentes patológicos, que fue sometida a una histerectomía y que tuvieron que reintervenir tres veces y recibir en total 14 unidades de eritrocitos, 16 de plaquetas y 8 de plasma fresco congelado, debido a los sangrados postoperatorios: durante su estancia en la unidad de cuidados intensivos, la paciente refirió que desde hacía seis meses ingería ocho dientes de ajo al día ${ }^{24}$. Luego, Lawn y Sains (2012), publicaron dos casos en los que también ocurrieron sangrados transoperatorios, en pacientes que refirieron automedicarse con un suplemento de ajo ${ }^{25}$. Por otra parte, el consumo de ajo induce el sistema del citocromo CYP 450, lo que incrementa el efecto de la warfarina, aspirina y clopidogrel que aumenta el riesgo de sangrados 8,13

Entonces, al lado de sus numerosas bondades, se debe tener cuidado con este riesgo de sangrado, por lo que se recomienda suspender la ingesta excesiva de ajo crudo o como suplemento, por lo menos siete días antes de una cirugía para evitar sangrados que podrían poner en riesgo la vida del paciente ${ }^{8,24}$. Por otro lado, es importante recalcar que el ajo posee más de 2000 sustancias bioactivas y la cantidad de estas varía dependiendo del tipo de presentación, ya sea ajo fresco, como aceite esencial, el método de extracción y el añejamiento. lo cual incrementa aún más las variables y el riesgo de interacciones farmacológicas ${ }^{14}$. Pero lo más importante, como señala Ernst, es evaluar científicamente si esos beneficios son reales in vivo, pues un metaanálisis mostró que sus ventajas eran modestas cuando se hacían estudios doble ciegos ${ }^{26}$.

\section{Hierba de San Juan (Hyperium perforatum)}

La hierba de San Juan es una planta perenne, que contiene más de doce componentes activos, de los cuales la hiperforina tiene propiedades antidepresivas, por inhibir la recaptación de neurotransmisores como la serotonina, noradrenalina, dopamina y glutamato ${ }^{27,28}$. En un estudio clínico se demostró que un extracto de la hierba de San Juan estandarizado de $350 \mathrm{mg}$, administrado tres veces al día, era más efectivo que el placebo y era equivalente a una dosis de $100 \mathrm{mg}$ de imipramina diaria, después de ocho semanas de uso en pacientes con depresión moderada ${ }^{29}$.

Sin embargo, se debe recalcar que la hierba de San Juan es un potente inductor del sistema de enzimas hepáticas CYP, principalmente de la $3 \mathrm{~A}^{4}$, la cual metaboliza aproximadamente el $50 \%$ de los medicamentos convencionales, lo que generaría una disminución en los niveles séricos del medicamento, por lo tanto, una submedicación y la necesidad de incrementar las dosis ${ }^{27,28,30}$. Por ejemplo, el uso de la hierba de San Juan en conjunto con la warfarina disminuye el efecto anticoagulante de esta última, lo cual incrementa el riesgo de producción de trombos en un paciente que ha sido medicado para evitar ese fin $^{28}$.

La administración de la hierba de San Juan y fármacos inhibidores selectivos de la recaptación de serotonina ha causado síntomas del síndrome serotonérgico, principalmente en adultos mayores, lo cual debe alertar a médicos pues dichos síntomas van desde la falta de coordinación, confusión, agitación, nauseas, rabdomiolisis hasta un estado de coma $^{28}$.

Por el contrario, varios de los constituyentes de la hierba de San Juan, inhiben otros de los sistemas hepáticos encargados del metabolismo, de modo que incrementan el efecto terapéutico, tal como ocurre cuando 


\section{Revista Electrónica Enfermeria Actual en costa Rica}

se administra esta hierba con tolbutamida, un hipoglicemiante, lo cual aumenta el riesgo de hipoglicemias ${ }^{28}$. Además, reduce las concentraciones sanguíneas de ciclosporina, amitriptilina, digoxina, indinavir, warfarina, teofilina y femprocumon, además de que se asocia a sangrado intermenstrual, delirio o síndrome de la serotonina leve, cuando se utiliza concomitantemente con anticonceptivos orales (etinilestradiol / desogestrel), loperamida o inhibidores selectivos de la recaptación de serotonina selectiva (sertralina, paroxetina, nefazodona) ${ }^{31}$.

Considerando lo anterior, el empleo de la hierba de San Juan debería notificarse al médico de cabecera, para que evalúe su utilización en el contexto médico del paciente, pues podría tener un efecto antagónico con el tratamiento preexistente; lo cual es muy importante, dado que se trata de un producto que puede adquirirse libremente en las tiendas de medicina natural, sin prescripción médica.

\section{Ginkgo biloba}

El ginkgo biloba, es el nombre científico de una planta que se ha utilizado tanto en la gastronomía como en la medicina tradicional orienta ${ }^{32}$. Actualmente, se comercializa un extracto registrado conocido como EGb761, que estandariza una formulación de gingko biloba, por medio de un procesamiento riguroso que inicia con la cosecha de solamente hojas verdes, las cuales son sometidas a un análisis morfológico para luego elaborar la extracción con acetona al $60 \%$ y evaluadas por medio de cromatografía de alta calidad ${ }^{32}$. Finalmente, el producto obtenido contiene aproximadamente $24 \%$ de glucósidos flavonoides, 2.6-3.4\% de ginkgolidos, los cuales tienen propiedades farmacológicas, 2.6-3.2\% de bilobalidos, también bioactivos y aseguran que el extracto tiene menos de $5 \mathrm{ppm}$ de ácido ginkgólico, pues es un alérgeno natural ${ }^{32,33}$. Aunque, aún no está aprobado por la Food and Drug Association de EEUU, es prescrito en Europa y utilizado en la mayoría de los ensayos clínicos por ser un producto herbario estandarizado ${ }^{32,34}$.

En un estudio realizado por Napryeyenko y colaboradores (2009) demostraron que el extracto de ginkgo biloba EGb761 es más efectivo que el placebo, para disminuir los síntomas neuropsiquiátricos y mejorar las habilidades para enfrentar la rutina diaria en pacientes con enfermedad de Alzheimer y con demencia vascular ${ }^{33}$. Asimismo, es utilizado como tratamiento en pacientes con demencia senil, daño cerebral traumático, arterosclerosis cerebral, edema cerebral entre otras afectaciones neurológicas, debido a que mejora el flujo sanguíneo cerebral y también actúa como antioxidante y como protector contra la disfunción mitocondrial ${ }^{34}$.

Sin embargo, el ginkgo biloba tiene propiedades antiplaquetarias, por ser un antagonista del factor activador de plaquetas e inhibidor de la agregación plaquetaria ${ }^{32}$, por lo cual incrementan los riesgos de sangrados, inclusive, hay casos reportados en la literatura en los cuales pacientes que han consumido esta hierba han tenido sangrados importantes. Rosenblatt y Mindel (1997) expusieron el caso de un paciente de 70 años que presentó visión borrosa y una coloración rojiza en la córnea, producto de la ingesta de 40mg ginkgo biloba dos veces al día y aspirina; sin embargo, al descontinuar solamente este producto herbario no volvió a presentar problemas $^{35}$. Asimismo, han ocurrido hemorragias espontáneas sin causa aparente, por el consumo de ginkgo biloba, las cuales se resuelven al cesar el consumo de este producto, por ende, debe alertarse al gremio de la salud, pues los pacientes podrían estar en riesgo de una complicación grave si estos sangrados no son resueltos oportunamente $^{36,37}$. 


\section{Revista Electrónica Enfermeria Actual en costa Rica}

\section{www.revenf.ucr.ac.cr}

\section{CONCLUSIONES}

Las hierbas ofrecen una alternativa para combatir diversas dolencias, tal como menciona Uriostegui ${ }^{1}$; en este mismo contexto, la ethnomedicina es una fuente potencial para la creación de nuevos medicamentos, inclusive es la esperanza para el descubrimiento de nuevos antimicrobianos para combatir infecciones causadas por microorganismos multiresistentes y otras enfermedades que actualmente carecen de un tratamiento definitivo como el cáncer y el SIDA, por mencionar un par de ejemplos. Sin embargo, es importante recalcar que al igual que cualquier fármaco, los medicamentos a base de hierbas generan efectos secundarios que podrían arriesgar la salud del paciente, ya sea por producir una interacción medicamentosa, por afectar directamente el proceso de coagulación u otros efectos. Da do lo anterior, el personal del área de la salud debe advertir a los pacientes sobre estos inconvenientes y ser muy enfático al preguntar al paciente sobre el consumo de algún producto natural, lo cual debe incluirse en la historia médica, pues con ello el profesional podrá evitar complicaciones luego de un tratamiento o durante el posoperatorio lo cual, a su vez, obliga al personal médico a investigar y actualizarse en cuanto a los riesgos e interacciones medicamentosas de las hierbas empleadas en medicina natural.

\section{REFERENCIAS BIBLIOGRÁFICAS}

1. Urióstegui-Flores A. Hierbas medicinales utilizadas en la atención de enfermedades del sistema digestivo en la ciudad de Taxco, Guerrero. Mexico. Rev Salud pública. [Internet] 2015; 17(1): 85-96. Disponible en: http://revistas.unal.edu.co/index.php/revsaludpublica/article/viewFile/42235/52961

2. Patwardhan B, Vaidya A, Chorghade M, Joshi S. Reverse Pharmacology and Systems Approaches for Drug Discovery and Development. Curr Bioact Compd. [Internet] 2008; 4(4):201-212. Disponible en: http://www.iaim.edu.in/pdf/CBC-RP-08.pdf

3. Wicks SM, Mahady GB. Herbal and Complementary Medicines Used for Women's Health. Medicines for Women. Switzerland: Springer International; 2015. p. 373-399.

4. Stickel F, Shouval D. Hepatotoxicity of herbal and dietary supplements: an update. Arch Toxicol. [Internet] 2015 Jun; 89(6):851-65. Disponible en: https://www.ncbi.nlm.nih.gov/pubmed/25680499

5. Posadzki P, Watson LK, Ernst E. Adverse effects of herbal medicines: an overview of systematic reviews. Clin Med (Lond). [Internet] 2013 Feb; 13(1):7-12. Disponible en:

https://www.ncbi.nlm.nih.gov/pubmed/23472485

6. $\quad$ Lipman, Timothy O. Herbal supplements. Curr Gastroenterol Rep. 2005; 7.4: 302-307.

7. Svetaz L, Zuljan F, Derita M, Petenatti E, Tamayo G, Cáceres A, et al. Value of the ethnomedical information for the discovery of plants with antifungal properties: A survey among seven Latin American countries. J Ethnopharmacol. [Internet] 2010; 127:137-158. Disponible en:

http://www.sciencedirect.com/science/article/pii/S0378874109005947?via\%3Dihub 


\section{Revista Electrónica Enfermeria Actual en costa Rica}

8. Gray S, West LM. Herbal medicines--a cautionary tale. N Z Dent J. [Internet] 2012 Jun; 108(2):6872. Disponible en: https://www.ncbi.nlm.nih.gov/pubmed/22788052

9. Phua DH, Zosel A, Heard K. Dietary supplements and herbal medicine toxicities—when to anticipate them and how to manage them. Int J Emerg Med.[Internet] 2009 Jun; 2(2): 69-76. Disponible en: https://www.ncbi.nlm.nih.gov/pmc/articles/PMC2700222/

10. Gupta D, Bleakley B, Gupta RK. Dragon's blood: Botany, chemistry and therapeutic uses. J Ethnopharmacol. [Internet] 2008; 115: 361-380. Disponible en: http://www.sciencedirect.com/science/article/pii/S0378874107005387?via\%3Dihub

11. Ernst E. A Scientist in Wonderland: A memoir of Searching for truth and Finding Trouble. Exeter UK: Imprint Academic; 2015. p. 173.

12. Picado Twight C. Obras completas Clodomiro Picado. [Internet] Vol. 6, Intervenciones del Dr. C. Picado T. en la prensa. Costa Rica: Editorial Tecnológica de Costa Rica; 1988. p. 444. Disponible en: http://revistas.tec.ac.cr/files/journals/10/librosgratis/ObrasClorito/OBRASCOMPLETASDECLODOMIROPICADOVOL6.pdf

13. Ried K, Fakler P. Potential of garlic (Allium sativum) in lowering high blood pressure: mechanisms of action and clinical relevance. Integr Blood Press Control. [Internet] 2014 Dec 9; 7:71-82. Disponible en: https://www.ncbi.nlm.nih.gov/pmc/articles/PMC4266250/

14. Gritsenko K, Muse I, Vydyanathan A. Herbal Supplements and Abuse. Substance Abuse. New York: Springer; 2015. p. 165-178.

15. Majewski M. Allium sativum: facts and myths regarding human health. Rocz Panstw Zakl Hig. [Internet] 2014; 65(1):1-8. Disponible en: https://www.ncbi.nlm.nih.gov/pubmed/24964572

16. Shridevi A, Raghavendra A, Kaushal S, Amol K. Garlic: Review of literature. Indian J Cancer. [Internet] 2014; 51(4): 577-581. Disponible en: http://www.indianjcancer.com/article.asp?issn=0019509X; year $=2014$; volume $=51 ;$ issue $=4$; spage $=577$; page $=581$; aulast $=$ Adaki

17. Seki T, Hosono T. Prevention of Cardiovascular Diseases by Garlic-Derived Sulfur Compounds. J Nutr Sci Vitaminol. [Internet] 2015; 61: S83-S85. Disponible en:

https://www.ncbi.nlm.nih.gov/pubmed/26598900

18. Borlinghaus J, Albrecht F, Gruhlke MCH, Nwachukwu ID, Slusarenko AJ. Allicin: Chemistry and Biological Properties. Molecules. [Internet] 2014; 19(8): 12591-12618. Disponible en: https://www.ncbi.nlm.nih.gov/pubmed/25153873 


\section{Revista Electrónica Enfermeria Actual en costa Rica}

19. Li G, Ma X, Deng L, Zhao X, Wei Y, Gao Z, et al. Fresh Garlic Extract Enhances the Antimicrobial Activities of Antibiotics on Resistant Strains in Vitro. Jundishapur J Microbiol. [Internet] 2015 May 31; 8(5):e14814. Disponible en: https://www.ncbi.nlm.nih.gov/pubmed/26060559

20. Cutler RR, Wilson P. Antibacterial activity of a new, stable, aqueous extract of allicin against methicillin resistant Staphylococcus aureus. Brit J Biomed Sci. [Internet] 2004; 61(2):71-74. Disponible en: https://www.ncbi.nlm.nih.gov/pubmed/15250668

21. Tsao SM, Liu WH, Yin MC. Two diallyl sulphides derived from garlic inhibit meticillin-resistant Staphylococcus aureus infection in diabetic mice. J Med Microbiol. [Internet] 2007 Jun; 56 (Pt 6):803-8. Disponible en: https://www.ncbi.nlm.nih.gov/pubmed/17510266

22. Karuppiah P, Rajaram S. Antibacterial effect of Allium sativum cloves and Zingiber officinale rhizomes against multiple-drug resistant clinical pathogens. Asian Pac J Trop Biomed. [Internet] 2012; 2(8):597-601. Disponible en: https://www.ncbi.nlm.nih.gov/pmc/articles/PMC3609356/

23. Robyn J, Rasschaert G, Hermans D, Pasmans F, Heyndrickx M. Is allicin able to reduce Campylobacter jejuni colonization in broilers when added to drinking water? Poult Sci. [Internet] 2013 May; 92(5):1408-18. Disponible en: Robyn J, Rasschaert G, Hermans D, Pasmans F, Heyndrickx M. Is allicin able to reduce Campylobacter jejuni colonization in broilers when added to drinking water? Poult Sci. 2013 May; 92(5):1408-18

24. Soleimani D, Paknahad Z, Askari G, Iraj B, Feizi A. Effect of garlic powder consumption on body composition in patients with nonalcoholic fatty liver disease: A randomized, double-blind, placebocontrolled trial. Adv. Biomed. Res. [Internet] 2016; 5:2. Disponible en:

https://www.ncbi.nlm.nih.gov/pubmed/26955623

25. Erian M, McLaren G. Be wary of "natural" therapy in gynecological surgery. Int J Womens Health. [Internet] 2013 Jun 20; 5:345-9. Disponible en: https://www.ncbi.nlm.nih.gov/pubmed/23843707

26. Lawn A, Sains P. Comment on: Not just a vampire repellent: the adverse effects of garlic supplements in surgery. Ann R Coll Surg Engl. [Internet] 2012 Sep; 94(6):451. Disponible: https://www.ncbi.nlm.nih.gov/pmc/articles/PMC3954349/

27. Max H. Pittler, Edzard Ernst Clinical effectiveness of garlic (Allium sativum). Mol Nutr Food Res [Internet] 2007; 51: 1382-5. Disponible en:

http://onlinelibrary.wiley.com/doi/10.1002/mnfr.200700073/abstract

28. Borrelli F, Izzo AA. Herb-drug interactions with St John's wort (Hypericum perforatum): an update on clinical observations. AAPS J. [Internet] 2009 Dec; 11(4):710-27. Disponible en: https://www.ncbi.nlm.nih.gov/pmc/articles/PMC2782080/ 


\section{Revista Electrónica Enfermeria Actual en costa Rica}

29. Zhou S, Chan E, Pan SQ, Huang M, Lee EJ. Pharmacokinetic interactions of drugs with St John's wort. J Psychopharmacol. [Internet] 2004 Jun; 18(2):262-76. Disponible en: http://journals.sagepub.com/doi/pdf/10.1177/0269881104042632

30. Philipp M, Kohnen R, Hiller KO. Hypericum extract versus imipramine or placebo in patients with moderate depression: randomised multicentre study of treatment for eight weeks. BMJ. [Internet] 1999; 319(7224):1534-1539. Disponible en: https://www.ncbi.nlm.nih.gov/pubmed/10591711

31. Biffignandi PM, Bilia AR. The growing knowledge of St. John's wort (Hypericum perforatum L) drug interactions and their clinical significance. Curr Therap Res. [Internet] 2000 Jul; 61(7): 389-394. Disponible en: http://www.currenttherapeuticres.com/article/S0011-393X(00)80021-1/pdf

32. Izzo AA, Ernst E. Interactions between herbal medicines and prescribed drugs: an updated systematic review. Drugs. [Internet] 2009; 69(13):1777-98. Disponible en: https://www.ncbi.nlm.nih.gov/pubmed/19719333

33. Nash KM, Shah ZA. Current Perspectives on the Beneficial Role of Ginkgo biloba in Neurological and Cerebrovascular Disorders. Integr Med Insights. [Internet] 2015 Nov 9; 10:1-9. Disponible en: https://www.ncbi.nlm.nih.gov/pubmed/26604665

34. Napryeyenko O, Sonnik G, Tartakovsky I. Efficacy and tolerability of Ginkgo biloba extract EGb 761 by type of dementia: analyses of a randomised controlled trial. J Neurol Sci. [Internet] 2009 Aug 15; 283(1-2):224-9. Disponible en: https://www.ncbi.nlm.nih.gov/pubmed/19286192

35. Diamond BJ, Bailey MR. Ginkgo biloba: indications, mechanisms, and safety. Psychiatr Clin North Am. [Internet] 2013 Mar; 36(1):73-83. Disponible en:

https://www.ncbi.nlm.nih.gov/pubmed/23538078

36. Rosenblatt M, Mindel J. Spontaneous hyphema associated with ingestion of Ginkgo biloba extract. N Engl J Med. [Internet] 1997 Apr 10; 336(15):1108. Disponible en: http://www.nejm.org/doi/full/10.1056/NEJM199704103361518

37. Carlile PV. Unexplained alveolar hemorrhage associated with Ginkgo and ginseng use. J Bronchology Interv Pulmonol. [Internet] 2015 Apr; 22(2):170-2. Disponible en: https://www.ncbi.nlm.nih.gov/pubmed/25887018

38. Bent S, Goldberg H, Padula A, Avins AL. Spontaneous Bleeding Associated with Ginkgo biloba: A Case Report and Systematic Review of the Literature. J Gen Intern. [Internet] 2005; 20(7):657-661. Disponible en: https://www.ncbi.nlm.nih.gov/pmc/articles/PMC1490168/ 